\title{
BMJ Open Secondhand tobacco smoke exposure among children under 5 years old: questionnaires versus cotinine biomarkers: a cohort study
}

\author{
Nerea Mourino (D) , ${ }^{1}$ Mónica Pérez-Ríos (D) , ${ }^{1,2}$ Maria Isolina Santiago-Pérez, ${ }^{2}$ \\ Bruce Lanphear, ${ }^{3}$ Kimberly Yolton, ${ }^{4}$ Joseph M Braun (i) ${ }^{5}$
}

To cite: Mourino N, Pérez-

Ríos M, Santiago-Pérez Ml, et al. Secondhand tobacco smoke exposure among children under 5 years old: questionnaires versus cotinine biomarkers: a cohort study. BMJ Open 2021;11:e044829. doi:10.1136/ bmjopen-2020-044829

- Prepublication history and supplemental material for this paper is available online. To view these files, please visit the journal online (http://dx.doi. org/10.1136/bmjopen-2020044829).

Received 21 September 2020 Accepted 03 June 2021

Check for updates

(c) Author(s) (or their employer(s)) 2021. Re-use permitted under CC BY-NC. No commercial re-use. See rights and permissions. Published by BMJ.

For numbered affiliations see end of article.

Correspondence to Dr Mónica Pérez-Ríos: monica.perez.rios@usc.es

\section{ABSTRACT}

Objectives Cotinine is the gold standard to estimate prevalence of secondhand tobacco smoke (SHS) exposure, and assay limit of detection (LOD) cut-points are typically used regardless of age. Our aim was to compare the concordance between mother-reported SHS exposure and serum cotinine categorising children as exposed with the assay LOD or age-specific cut-points.

Design Data from the Health Outcomes and Measures of the Environment (HOME) Study, a prospective pregnancy and birth cohort.

Setting Hospital or participants' homes.

Participants 389 pregnant women aged 18 years and older, between 13 and 19 weeks of gestation, living in a five-county region of the Cincinnati, Ohio metropolitan area, and with follow-up on their children at birth and ages 12, 24, 36 and 48 months.

Primary and secondary outcome measures Children's serum cotinine, mother-reported active smoking and SHS exposure were available at birth and during followup visits. We used Cohen's kappa index to assess concordance between maternal self-report and child's serum cotinine concentrations. We estimated optimal age-specific cut-points, their sensitivity-specificity and positive-negative predictive values with receiver operating characteristic curves.

Results Self-reported exposure and cotinine data were available for 280 women who gave birth to singleton child. When applying the assay LOD $(0.015 \mathrm{ng} / \mathrm{mL})$, concordance between maternal report and serum cotinine, without accounting for age, was below 0.23 at all times. When using age-specific cut-points (12 months: $0.11 \mathrm{ng} / \mathrm{mL}$; 24 months: $0.08 \mathrm{ng} / \mathrm{mL} ; 36$ months: $0.05 \mathrm{ng} / \mathrm{mL}$ and 48 months: $0.04 \mathrm{ng} / \mathrm{mL}$ ), concordance improved, being low at 12 months $(0.39)$, moderate at 24 and 36 months $(0.47$ and 0.43 ) and high at 48 months (0.62).

Conclusions Concordance between mother-reported SHS exposure among children under 5 years and serum cotinine improved considerably after applying the cohortspecific and age-specific cut-points. Future studies are necessary to verify these results.

\section{BACKGROUND}

Exposure to secondhand tobacco smoke (SHS), or passive smoking, is the involuntary
Strengths and limitations of this study

- First study estimating serum cotinine age-specific cut-points for children under 5 years old.

- This study has one of the largest samples of children younger than 5 years to validate secondhand tobacco smoke (SHS) exposure using parental report and biomarkers.

- We have longitudinal measures of SHS exposure derived from both maternal report and children's serum cotinine concentrations over the first 4 years of life.

- We do not include questions to evaluate third-hand smoke exposure or dietary intake.

- We use concordance as another way of validating the discriminatory capacity of the receiver operating characteristic curve.

inhalation of a complex mixture of tobacco smoke produced by the consumption of tobacco. ${ }^{1}$ SHS exposure is a global public health concern, and there is no safe threshold of exposure. ${ }^{2}$ Children are especially vulnerable to the effects of SHS exposure due to their narrower airways, faster respiratory rate and undeveloped immune system. ${ }^{3}$ The detrimental effects of SHS exposure on children's health, which have been documented since the 1970s, include an increased risk of sudden infant death syndrome, acute respiratory tract infections (bronchitis and pneumonia), asthma exacerbation, respiratory symptoms (cough, phlegm, wheeze and breathlessness) and ear infections. ${ }^{4}$ Over the last 50 years, more than 100000 infants exposed to SHS have already died in the USA. ${ }^{5}$

Parent-reported SHS exposure, which is often used to estimate SHS exposure, suffers from recall bias, social desirability bias and lack of knowledge about the child's exposure in the parents' absence. ${ }^{6}$ As a result, having access to sensitive and specific biomarkers of 
SHS exposure, such as cotinine, is generally considered a more valid and reliable method for estimating exposure. ${ }^{1}$ Cotinine, which is the primary metabolite of nicotine, is considered the optimal biomarker for measuring SHS exposure. Cotinine has high specificity and sensitivity, ${ }^{7}$ as well as a prolonged half-life, relative to nicotine, which ranges from 16 to 20 hours in children. ${ }^{1}$ Cotinine can be quantified in serum, urine, hair, saliva, maternal milk, amniotic fluid and meconium. ${ }^{89}$ While there are validated cotinine cut-points that can differentiate between active smokers and non-smokers among adults, such cut-points are not well established for distinguishing SHS exposure among children. ${ }^{10-12}$ Most studies use the analytical technique limit of detection (LOD) to define SHS exposure.

Various investigators, who have evaluated the validity of information collected from questionnaires using cotinine as the gold standard, have found that the concordance between parental self-report and cotinine measured in child's serum is inconsistent. ${ }^{13-16}$ These inconsistencies could be related to poor validity of parental self-report, age-related differences or a lack of adequate cut-points for cotinine concentrations among children. ${ }^{17}{ }^{18}$ Given that assays for cotinine are so sensitive, the values above the LOD might be derived from other sources of nicotine, such as diet. ${ }^{719}$ Thus, the establishment of valid cut-points for distinguishing SHS exposure from non-exposure among children could reduce exposure misclassification.

The purpose of this study was to characterise the concordance between mother-reported SHS exposure and serum cotinine concentrations in children younger than 5 years and assess the utility of age-specific serum cotinine cut-points to characterise children's SHS exposure compared with the serum cotinine assay LOD.

\section{METHODS}

\section{Study participants}

We used data from the Health Outcomes and Measures of the Environment (HOME) Study, a prospective cohort study that enrolled pregnant women from the Cincinnati, Ohio, from 2003 to $2006 .{ }^{20}$ The principal objective of the HOME Study was to evaluate the association of prenatal and postnatal exposure to environmental toxicants with health and neurobehavioural outcomes in infants and children. The inclusion criteria were: $\geq 18$ years old, between 13 and 19 weeks of gestation, residing in a house built before 1978 within the study area, HIV negative, not taking thyroid or epilepsy medication and not undergoing chemotherapy or radiation therapy. From March 2003 to January 2006, we recruited 468 pregnant women living in a five-county region of the Cincinnati, Ohio metropolitan area (Butler, Clermont, Hamilton and Warren counties) and Northern Kentucky (Campbell county) to participate in a longitudinal pregnancy and birth cohort study. Sixty-seven women dropped out in pregnancy during the run-in phase of a randomised controlled trial of residential lead and injury hazard controls nested within the cohort. From 2003 to 2014, we conducted up to 11 in-person follow-up visits on 410 eligible children (390 singleton and 10 twin sets) at the delivery hospital, our study clinic or participants' homes when children were approximately 1 day, 4 weeks and 1, 2, 3, 4, 5 and 8 years of age; follow-up rates ranged from $94 \%$ (age 4 weeks) to $48 \%$ (age 4 years). A detailed description of the cohort is published elsewhere. ${ }^{20}$

\section{Patient and public involvement}

A community advisory board provided feedback on the original design of the HOME Study before the study began. Before initiating any new follow-up, we conducted pilot testing to ensure that the visit length and types of assessments were appropriate. At more recent childhood follow-up visits, we collected information regarding the visit length and experience from participants and used this to inform the development of subsequent visits. We previously reported back concentrations of environmental chemical biomarkers to participants while also providing contextual information. Finally, we reported clinically significant findings to participants and their medical providers.

\section{Assessment of SHS exposure}

Maternal-reported tobacco consumption and SHS exposure

Maternal-reported tobacco consumption and children's SHS exposure were obtained by using standardised faceto-face interviews administered by a trained interviewer. The questionnaire was administered during pregnancy and at five different points during the follow-up: 4 weeks after birth and when children were ages 12, 24, 36 and 48 months. At each interview, trained research staff surveyed the women about their smoking of cigarettes, cigars and pipes as well as the smoking of these products by other members of the household. Women were also asked about their SHS exposure and that of their child at home (living with a smoker who smokes at home), in other frequently visited homes and in the car. Each mother was classified as either a smoker, exposed (non-smoker with SHS exposure) or unexposed (non-smoker with no SHS exposure). Each child was classified as exposed if the mother reported either being a smoker or living with a smoker who smokes at home or if the mother reported that her child was exposed to SHS in the car or in other homes and places (such as grandmother's home or daycare). Otherwise, we classified children as unexposed.

\section{Serum biomarkers of SHS exposure}

We collected venous serum samples from children at delivery (umbilical cord) and at 12, 24, 36 and 48 months via venipuncture. The samples were stored at or below $-80^{\circ} \mathrm{C}$ until analysis. Serum cotinine concentrations were determined by the Centers for Disease Control and Prevention Environmental Health Laboratories using high performance liquid chromatography atmospheric pressure tandem mass spectrometry. The assay LOD threshold for cotinine was $0.015 \mathrm{ng} / \mathrm{mL} .{ }^{19}$ We classified women as unexposed if the cotinine concentration from 
newborns' umbilical cord blood after birth was $<$ LOD, SHS exposed if the value was $\geq \mathrm{LOD}$ but $\leq 3 \mathrm{ng} / \mathrm{mL}$ and smokers if the cotinine concentration was $>3 \mathrm{ng} / \mathrm{mL}{ }^{11}$ Children were classified as unexposed if their cotinine concentration was $<$ LOD and exposed if it was $\geq$ LOD. After calculating age-specific cut-points for children (described further down), we classified them as unexposed if their cotinine concentration was lower than the new cut-points and exposed if it was equal or higher than the new cut-points.

\section{Statistical analysis}

We calculated descriptive analysis for maternal sociodemographic characteristics, serum cotinine concentrations and SHS exposure prevalence in children using maternal-reported information and serum cotinine concentrations considering both assay LOD derived cut-point and age-specific cut-points. The concordance between maternal-reported SHS exposure and categories of serum cotinine concentrations were calculated using Cohen's kappa index for two observers, considering two categories (exposed/unexposed). Correlation and agreement between children's log-base transformed serum cotinine concentrations at different moments between 12 and 48 months of age were estimated using Pearson correlation coefficients $(r)$ and intraclass correlation coefficient (ICC).

We used receiver operating characteristic (ROC) curves to identify the optimal serum cotinine concentration to distinguish SHS exposure from non-exposure in children at 12, 24, 36 and 48 months. For this calculation, we considered several information reported by mothers regarding their children's exposure to SHS to validate the discriminatory capacity of the ROC curve. Exposed category comprised children whose mothers reported either being a smoker or living with a smoker who smokes at home and those whose mothers reported they were exposed in the car or in other homes and places (such as grandmother's home or daycare). Area under ROC curves (AUCs) was calculated besides age-specific cutpoints. There are various methods to optimise cotinine cut-points. We tested the three criteria most often used in biostatistics: maximising the Youden index, the identification of the point on the curve with minimum distance from the left-upper corner of the unit square and minimising the difference between sensitivity and specificity; while the three methods provided similar results, we chose the third approach to be able to identify the optimal agespecific cut-point that maximises both sensitivity and specificity, to minimise misclassification either as exposed or unexposed based on cotinine concentrations. Specificitysensitivity and positive-negative predictive values (PPVNPV) were calculated for each age-specific cut-point. Estimations were accompanied by confidence intervals of $95 \%$ (95\% CI). Analysis is restricted to children with mother-reported information and serum sample. The analysis was performed by using Stata V.14.2.
RESULTS

\section{Sample characteristics}

A total of 384 women had complete data on their tobacco smoke exposure at delivery, while $336(87.5 \%)$ children had complete self-report data at 12 months, $280(72.9 \%)$ at 24 months, $258(67.2 \%)$ at 36 months and $187(48.7 \%)$ at 48 months. At baseline, $31 \%$ of women were between ages 30 and 34 years, $62 \%$ were non-Hispanic white, $75 \%$ had greater than high school education, $81 \%$ were employed, $78 \%$ lived with a spouse or partner and $71 \%$ had private health insurance. The attrition rate of women from pregnancy to the 48-month age period was 51.3\%; loss to follow-up was not related to any sociodemographic characteristics (online supplemental table 1).

We restricted the analyses to children with both maternal self-reported tobacco consumption and SHS exposure information and cotinine measures. Information was available for 280 newborns, 270 children at 12 months, 197 at 24 months, 196 at 36 months and 150 at 48 months (online supplemental table 2 ). The attrition rate of these children with both self-reported data and serum biomarkers of SHS exposure was $46.4 \%$ from delivery to age 48 months (online supplemental table 1 ).

\section{Serum cotinine distribution}

Children's geometric mean (GM) serum cotinine concentrations from 12 to 48 months was higher than newborns' GM umbilical cord serum concentrations (table 1). Serial measures of children's serum cotinine concentrations from 12 to 48 months were highly correlated with correlation coefficients between log-transformed children's serum cotinine concentrations in consecutive periods ranging from 0.81 (24-36 months) to 0.72 (12-36 months). The ICC between repeated serum cotinine concentrations (analysis restricted to 71 children with cotinine measures at 12, 24, 36 and 48 months) was 0.72 (95\% CI 0.63 to 0.80 ) reflecting good agreement between measurements.

\section{Prevalence of exposure to SHS}

The prevalence of children exposed to SHS based on maternal report varied between $26.8 \%$ and $31.3 \%$ (figure 1). The prevalence of SHS exposure based on cord serum cotinine after applying the assay LOD derived cut-point of $0.015 \mathrm{ng} / \mathrm{mL}$ was double the self-reported prevalence. The prevalence of SHS exposure based on children's serum cotinine concentrations decreased from $86.7 \%$ at 12 months to $74.7 \%$ at 48 months (figure 1 upper). The difference between maternal-reported prevalence of exposure and that estimated from children's serum cotinine concentrations, excluding newborns and using LOD as cut-point, was nearly 50 percentage points at any age.

Children whose mothers reported SHS exposure had higher serum cotinine concentrations than children whose mothers reported no exposure to SHS (figure 2). Fifty per cent of the newborns born to self-reported smokers had cord serum cotinine concentrations $>3 \mathrm{ng} /$ 
Table 1 Descriptive statistics of serum cotinine concentrations $(\mathrm{ng} / \mathrm{mL})$ at birth and at $12,24,36$ and 48 months: $\mathrm{N}$, range, quartiles and geometric mean with $95 \% \mathrm{Cl}$

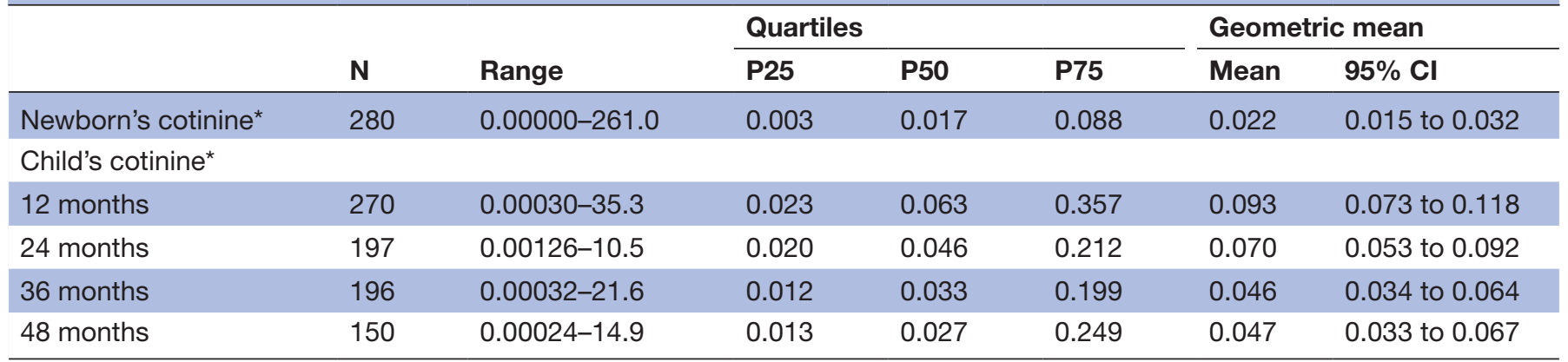

*Analysis is restricted to participants with both maternal-reported data and cotinine measures.

$\mathrm{N}$, number of observations; $\mathrm{P}$, percentile.

mL. At 12 and 24 months of age, $83 \%$ and $80 \%$ of children whose mothers reported that they were not exposed had cotinine values higher than the LOD, and at 36 and 48 months of age, the percentage was $66 \%$ and $65 \%$. Moreover, the distribution of serum cotinine concentration was similar among children whose mothers were active smokers and non-smoking mothers who reported SHS exposure (figure 2); for this reason, these categories were combined in further analysis.

\section{Estimation of age-specific cut-points for distinguishing SHS exposure}

The AUC of various serum cotinine thresholds ranged from 0.80 and 0.89 (online supplemental figure 1). Cut-points for distinguishing SHS exposure from nonexposure decreased with child age and were set at 0.11

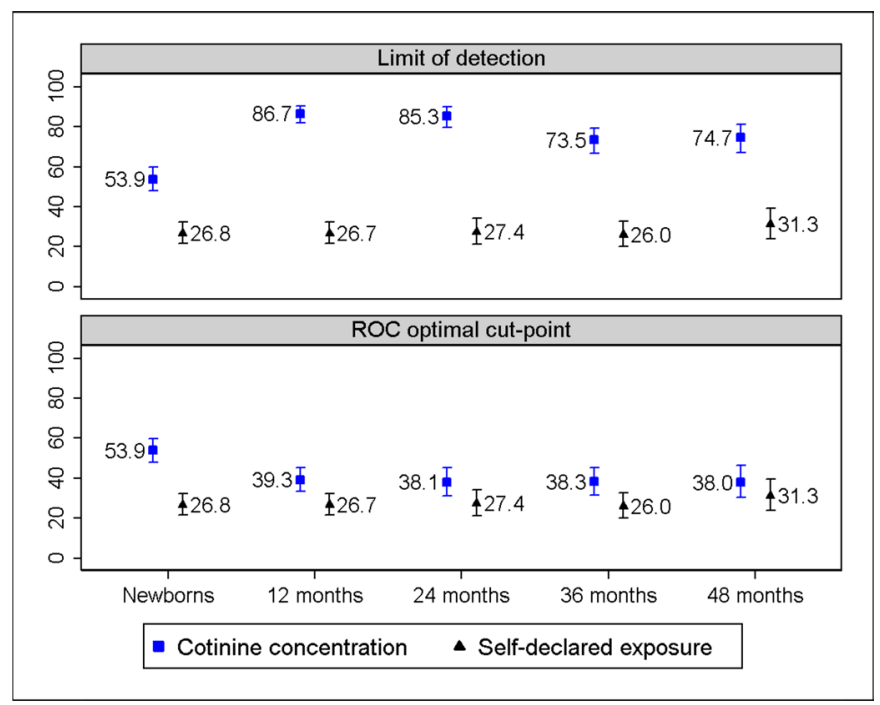

Figure 1 Prevalence of SHS exposure among children is derived from maternal self-report (exposed/unexposed), depicted with a triangle, and also from serum cotinine concentrations, depicted with a square, applying assay LOD derived cut-point of $0.015 \mathrm{ng} / \mathrm{mL}$ (upper) and age-specific cut-points of $0.11 \mathrm{ng} / \mathrm{mL}$ at 12 months; $0.08 \mathrm{ng} / \mathrm{mL}$ at 24 months; $0.05 \mathrm{ng} / \mathrm{mL}$ at 36 months; and $0.04 \mathrm{ng} / \mathrm{mL}$ at 48 months (bottom). LOD, limit of detection; ROC, receiver operating characteristic; SHS, secondhand tobacco smoke. $\mathrm{ng} / \mathrm{mL}$ at 12 months, $0.08 \mathrm{ng} / \mathrm{mL}$ at $24,0.05 \mathrm{ng} / \mathrm{mL}$ at 36 and at $0.04 \mathrm{ng} / \mathrm{mL}$ at 48 months (table 2). The sensitivity and specificity corresponding to these cutpoints were above $72 \%$, and NPV was over $87 \%$. Using the optimal serum cotinine age-specific cut-points, the prevalence of SHS was highest at 12 months (39.3\%) and

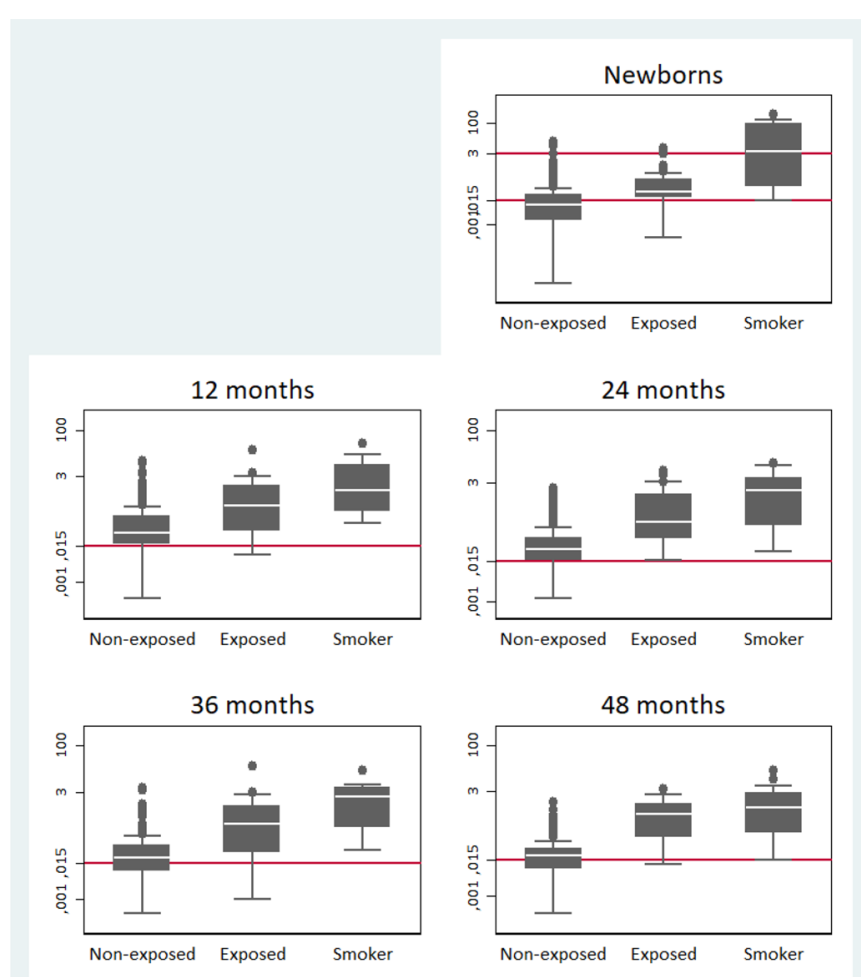

Figure 2 The box plots depict the distribution of serum cotinine concentrations $(\mathrm{ng} / \mathrm{mL})$, as logarithm, from neonatal umbilical cord (upper line $=3 \mathrm{ng} / \mathrm{mL}$ and bottom line $=0.015$ $\mathrm{ng} / \mathrm{mL}$ ) and child at 12, 24, 36 and 48 months (line= 0.015 $\mathrm{ng} / \mathrm{mL}$ ) depending on children's SHS exposure reported by mothers (unexposed/exposed/mother smoker). If using the LOD derived cut-point of $0.015 \mathrm{ng} / \mathrm{mL}$ to distinguish between SHS exposure/non-exposure, all the children, including those from the non-exposure category, had serum cotinine concentrations comparable with SHS exposure. LOD, limit of detection; SHS, secondhand tobacco smoke. 
Table 2 AUC, new age-specific cut-points for each age $(\mathrm{ng} / \mathrm{mL})$ with its sensitivity, specificity, PPV and NPV

\begin{tabular}{lllll}
\hline & $\mathbf{1 2}$ months & 24 months & $\mathbf{3 6}$ months & $\mathbf{4 8 ~ m o n t h s ~}$ \\
\hline $\mathrm{n}$ & 270 & 197 & 196 & 150 \\
Exposed to SHS, $\mathrm{n}(\%)$ & $72(26.7)$ & $54(27.4)$ & $51(26.0)$ & $47(31.3)$ \\
\hline AUC $(95 \% \mathrm{Cl})$ & $0.80(0.74$ to 0.86$)$ & $0.83(0.76$ to 0.90$)$ & $0.84(0.77$ to 0.91$)$ & $0.89(0.82$ to 0.95$)$ \\
\hline Cut-points $(\mathrm{ng} / \mathrm{mL})^{\star}$ & $\mathbf{0 . 1 1}$ & $\mathbf{0 . 0 8}$ & $\mathbf{0 . 0 5}$ & $\mathbf{0 . 0 4}$ \\
\hline Sensitivity $(95 \% \mathrm{Cl})$ & $72.20(60.40$ to 82.10$)$ & $75.90(62.40$ to 86.50$)$ & $74.50(60.40$ to 85.70$)$ & $83.00(69.20$ to 92.40$)$ \\
Specificity $(95 \% \mathrm{Cl})$ & $72.70(66.00$ to 78.80$)$ & $76.20(68.40$ to 82.90$)$ & $74.50(66.60$ to 81.40$)$ & $82.50(73.80$ to 89.30$)$ \\
PPV $(95 \% \mathrm{Cl})$ & $49.10(39.20$ to 59.00$)$ & $54.70(42.70$ to 66.20$)$ & $50.70(38.90$ to 62.40$)$ & $68.40(54.80$ to 80.10$)$ \\
\hline NPV $(95 \% \mathrm{Cl})$ & $87.80(81.80$ to 92.40$)$ & $89.30(82.50$ to 94.20$)$ & $89.30(82.30$ to 94.20$)$ & $91.40(83.80$ to 96.20$)$ \\
\hline
\end{tabular}

Estimations are accompanied by $95 \% \mathrm{Cl}$.

${ }^{*}$ Age-specific cut-points (bold values) are those that maximised the AUC, that is to say, those that minimise the difference between sensitivity and specificity. These values were calculated with receiver operating characteristic curves and children's SHS exposure reported by their mothers was considered the gold standard. Children's serum cotinine concentrations above these cut-point values will reflect SHS exposure. AUC, area under receiver operating characteristic curves; NPV, negative predictive value; PPV, positive predictive value; SHS, secondhand tobacco smoke.

lowest at 48 months $(38.0 \%)$. The greatest difference between maternal self-reported prevalence and serum cotinine estimated prevalence of SHS exposure is nearly 13 percentage points at 12 months (figure 1 bottom).

\section{Concordance between self-reported exposure and serum cotinine measures}

The concordance between maternal-reported SHS exposure and serum cotinine improved considerably after applying age-specific cut-points. The kappa coefficient between mother-reported exposure and child's serum cotinine concentrations, using the LOD as threshold, was below 0.22 in each of the four time periods. In contrast, when age-specific cut-points were used, the kappa coefficient improved from 0.39 at 12 months to 0.62 at 48 months (table 3). Taking Landis and Koch criteria into account in assessing the kappa index, when using the assay LOD of $0.015 \mathrm{ng} / \mathrm{mL}$ as cut-point, the concordance between maternal-reported and children's serum cotinine concentrations after delivery was insignificant at 12, 24 and 36 months and low at 48 months. When using the new age-specific cut-points, concordance improved with age, being low at 12 months, but moderate at 24, 36 and high at 48 months.

\section{DISCUSSION}

In this cohort, preschool-aged children whose mothers reported SHS exposure had higher serum cotinine concentrations than children whose mothers reported no SHS exposure. When using the serum cotinine assay LOD as the threshold for distinguishing SHS exposure from no exposure, concordance between serum cotinine concentrations and maternal-reported exposure was nonsignificant. In contrast, after deriving age-specific serum cotinine cut-points, the concordance between serum cotinine and mother-reported exposure to SHS improved, with increasing concordance as child age increased.

Various studies conclude that serum cotinine concentrations in children vary as a function of age, sex or race. $^{2}{ }^{21} 22$ With respect to age, children seem to have

Table 3 Kappa concordance coefficient between maternal-reported SHS exposure (exposed/unexposed) and child's serum cotinine concentrations accompanied with the percentage of agreement when using the assay LOD threshold and age-specific cut-points at $12,24,36$ and 48 months of age

\begin{tabular}{|c|c|c|c|c|}
\hline & 12 months & 24 months & 36 months & 48 months \\
\hline \multicolumn{5}{|c|}{ Assay LOD threshold* } \\
\hline Agreement (\%) & 38.52 & 42.13 & 49.49 & 54.00 \\
\hline Kappa $(95 \%$ Cl) & 0.08 (0.04 to 0.13$)$ & $0.12(0.07$ to 0.17$)$ & $0.18(0.10$ to 0.25$)$ & 0.22 (0.13 to 0.32$)$ \\
\hline \multicolumn{5}{|c|}{ Age-specific cut-points $†$} \\
\hline Agreement (\%) & 72.59 & 76.14 & 74.49 & 82.67 \\
\hline Kappa (95\% Cl) & 0.39 (0.28 to 0.50$)$ & 0.47 (0.34 to 0.59$)$ & $0.43(0.30$ to 0.56$)$ & 0.62 (0.49 to 0.75$)$ \\
\hline
\end{tabular}

${ }^{*}$ Assay LOD threshold to discriminate between children exposed and unexposed to SHS: $0.015 \mathrm{ng} / \mathrm{mL}$.

†Age-specific cut-points $(\mathrm{ng} / \mathrm{mL})$ to discriminate between children exposed and unexposed to SHS calculated with receiver operating characteristic curves, 12 months: $0.11 ; 24$ months: 0.08; 36 months: 0.05; 48 months: 0.04.

LOD, limit of detection; SHS, secondhand tobacco smoke. 
higher cotinine concentrations than adults at similar exposures. This could be due to metabolic differences, or to the fact that children have a faster respiration rate and inhale larger quantities of SHS contaminants than adults. ${ }^{23}$ Previous studies found differences in cotinine concentrations among children in different age groups, with higher concentrations among the youngest children. ${ }^{12}{ }^{24}$ Our results are consistent with this as GM serum cotinine concentrations increased from birth to 12 months and then declined again, possibly reflecting decreased respiratory rates as children aged. Differences between cord blood and later concentrations could be explained by the higher metabolism and faster elimination of cotinine in their mothers. ${ }^{25}$ Thus, results of prior studies and developmental appropriate changes in child behaviour, anatomy and physiology support the need for age-specific cut-points for children. Indeed, when the serum cotinine assay LOD threshold was used to distinguish SHS exposure, $65 \%-83 \%$ of children classified as unexposed by mother report were reclassified as SHS exposed. In contrast, when we used the new age-specific cut-points, only $17 \%-27 \%$ were reclassified as SHS exposed. Our results (data not shown) show that among the children whose mothers declared that they were not smokers at the four follow-up periods and that the children were not exposed to SHS, the cotinine concentration decreased as age increased. This decrease is unlikely to be due to misclassification of self-reported SHS by the mother.

Other investigators have concluded that the prevalence of SHS exposure obtained from maternal-report consistently underestimates actual exposure. Presumably, some of this estimate is because mothers might not report SHS exposure because of recall bias, social desirability bias or ignorance about their children's exposure in other settings. ${ }^{12}{ }^{21}$ Children's exposure may be so negligible that mothers are not able to identify or quantify it. ${ }^{6}$ Our results indicate that some of the discordance was because of failure to account for age-related differences in exposure or metabolism.

Based on cotinine derived from the LOD threshold, the prevalence of SHS exposure was $73.5 \%$ and $74.7 \%$ at 3 and 4 years old, respectively. Taking into account the estimates obtained in population studies, about 4 out of 10 US children aged $3-11$ years $(40.6 \%)$ are exposed to secondhand smoke. ${ }^{2}$ These large differences, however, fail to assess the influence of other factors such as the sensitivity and suitability of the cut-points used to classify exposure to SHS. The assay LOD has become much more sensitive over time. Earlier studies set it at $0.05 \mathrm{ng} /$ $\mathrm{mL},{ }^{41217}$ while more recent studies, including ours, have a LOD of $0.015 \mathrm{ng} / \mathrm{mL}$. This lower LOD could mean that low serum cotinine concentrations reflect transient SHS exposure or exposure from other sources, such as food (tomatoes, potatoes, cauliflower and black tea). Food consumption levels of dietary nicotine are insignificant compared with moderate SHS exposure, but consumption of high quantities of nicotine-containing foodstuffs might contribute to low-level elevations in serum cotinine (eg, $80 \mathrm{~g}$ of eggplant is equivalent to approximately $0.01 \mathrm{ng} / \mathrm{mL}$ of serum cotinine) ${ }^{7}$ Future studies could verify this hypothesis using studies with detailed dietary information and sensitive cotinine biomarkers.

Despite the improvement in the concordance between maternal-reported SHS and children's serum cotinine concentrations using the age-specific cut-points, misclassification is still a problem. This misclassification has been observed in prior studies, including those of pregnant women and their children younger than 5 years, ${ }^{26-28}$ and it could be due to maternal concealment to avoid social judgement or ignorance about negligible and transient low-level nicotine exposures quantified with sensitive cut-points.

This study has some limitations worth nothing. First, we had a modest sample size. Still, it was one of the largest samples of children younger than 5 years to validate SHS exposure using parental report and biomarkers. ${ }^{12} 272930$ Another limitation is the attrition of study participants. Yet loss to follow-up was not associated with any measured sociodemographic characteristic. Third, we did not include questions to evaluate third-hand smoke exposure, such as involuntary inhalation or cutaneous absorption of nicotine particles deposited on clothing and furniture, or dietary intake. Fourth, we lacked information about the duration of exposure to SHS, which could have been used to provide more valid and reliable cotinine thresholds, and we did not account for factors such as the size of the home, the intensity of exposure or the proximity to smokers. ${ }^{73} 32$ Fifth, to identify the optimal serum cotinine cut-points, we assumed maternal report was the gold standard. We note that we expect any misreporting to predominately affect the sensitivity of maternal report and not the specificity; few women would report exposure in its absence. It should be noted that maternal self-report does not refer solely to whether the mother declares that the child is exposed, but it also takes into consideration if the mother is a smoker and if the child lives with other smokers who smoke at home. Finally, our findings may not be generalisable to other populations as our eligibility criteria were not designed to ensure that our cohort was representative of births in the study region. However, most chemical biomarker concentrations among HOME Study participants are similar to pregnant women and children in the USA during the time of enrolment and follow-up. ${ }^{20}$

This study also has several strengths. First, we had longitudinal measures of SHS exposure derived from both maternal report and children's serum cotinine concentrations over the first 4 years of life. Second, our cohort was relatively higher SES, with $75.2 \%$ of the mothers having greater than high school education. Thus, misreporting of SHS exposure is likely reduced as previous studies have shown that higher educational level is associated with more accurate SHS exposure reporting. ${ }^{15}$ 


\section{CONCLUSIONS}

Previous investigators have concluded that maternal reports dramatically underestimate children's SHS exposure. When we used age-specific cut-points, we found that many fewer children were reclassified as SHS exposed. Thus, maternal report may be a better indicator of children's SHS than previous estimates. The age specific cutpoints should be validated in other cohorts.

\section{Author affiliations}

${ }^{1}$ Department of Preventive Medicine and Public Health, University of Santiago de Compostela, Santiago de Compostela, Spain

${ }^{2}$ Epidemiology Unit, Xunta de Galicia, Santiago de Compostela, Spain

${ }^{3}$ Department of Health Sciences, Simon Fraser University - Vancouver, Vancouver, British Columbia, Canada

${ }^{4}$ Department of Pediatrics, Cincinnati Children's Hospital Medical Center, Cincinnati, Ohio, USA

${ }^{5}$ Department of Epidemiology, Brown University, Providence, Rhode Island, USA

Acknowledgements We would like to thank the Health Outcomes and Measures of the Environment (HOME) Study staff, patient advisers and participants for their years of dedication to the study.

Contributors MP-R, MIS-P and JMB planned the study and supervise it with contribution from all the other authors. MP-R and MIS-P performed the analysis with substantive contribution from all the other authors, MP-R and NM wrote the first draft of the manuscript and MIS-P, JMB, BL and KY provided critical comments and contributed on the drafts to successive versions of the manuscript. All the authors approved the final version paper. MP-R is the guarantor.

Funding The HOME Study was funded by National Institutes of Environmental Health Sciences grants P01 ES011261, R01 ES014575 and R01 ES015517. The US National Institutes (National Institute of Environmental Health Sciences), Environmental Protection Agency, Department of Housing and Urban Development and Flight Attendant Medical Research Institute provided funding for the presented results in the HOME Study.

Competing interests JMB received an honoraria from Quest Diagnostic for serving on an expert panel related to endocrine disrupting chemicals. JMB's institution was financially compensated for his services as an expert witness for plaintiffs in litigation related to per- and polyfluoroalkyl (PFAS)-contaminated drinking water; these funds were not paid to JMB directly.

Patient consent for publication Not required.

Ethics approval The institutional review boards of Cincinnati Children's Hospital Medical Centre, the participating delivery hospitals and the Centers for Disease Control and Prevention approved this study. During face-to-face visit, research assistants explained study protocols to each prospective participant and completed a checklist to ensure women were fully informed about the study. All mothers provided written informed consent for both themselves and their children prior to enrolment. ID of approval: 01-8-5.

Provenance and peer review Not commissioned; externally peer reviewed.

Data availability statement Data are available on reasonable request. The HOME Study principal investigators welcome new collaborations with other investigators and have actively engaged in collaborative data sharing projects. Interested investigators should contact Drs Joseph M Braun (joseph_braun_1@brown.edu) and Kimberly Yolton (kimberly.yolton@cchmc.org) to obtain additional information about The HOME Study, discuss collaborative opportunities and request a project proposal form. The HOME Study Protocol Review Committee reviews proposed research projects to ensure that they do not overlap with extant projects and are an efficient use of scarce resources (eg, biospecimens).

Supplemental material This content has been supplied by the author(s). It has not been vetted by BMJ Publishing Group Limited (BMJ) and may not have been peer-reviewed. Any opinions or recommendations discussed are solely those of the author(s) and are not endorsed by BMJ. BMJ disclaims all liability and responsibility arising from any reliance placed on the content. Where the content includes any translated material, BMJ does not warrant the accuracy and reliability of the translations (including but not limited to local regulations, clinical guidelines, terminology, drug names and drug dosages), and is not responsible for any error and/or omissions arising from translation and adaptation or otherwise.
Open access This is an open access article distributed in accordance with the Creative Commons Attribution Non Commercial (CC BY-NC 4.0) license, which permits others to distribute, remix, adapt, build upon this work non-commercially, and license their derivative works on different terms, provided the original work is properly cited, appropriate credit is given, any changes made indicated, and the use is non-commercial. See: http://creativecommons.org/licenses/by-nc/4.0/.

\section{ORCID iDs}

Nerea Mourino http://orcid.org/0000-0003-2008-5913

Mónica Pérez-Ríos http://orcid.org/0000-0003-4456-6631

Joseph M Braun http://orcid.org/0000-0002-5239-3235

\section{REFERENCES}

1 Florescu A, Ferrence R, Einarson T, et al. Methods for quantification of exposure to cigarette smoking and environmental tobacco smoke: focus on developmental toxicology. Ther Drug Monit 2009;31:14-30.

2 Homa DM, Neff LJ, King BA, et al. Vital signs: disparities in nonsmokers' exposure to secondhand smoke--United States, 19992012. MMWR Morb Mortal Wkly Rep 2015;64:103-8.

3 Hang B, Wang P, Zhao Y, et al. Adverse health effects of Thirdhand smoke: from cell to animal models. Int J Mol Sci 2017;18:932.

4 U.S. Department of Health and Human Services. The health consequences of involuntary exposure to tobacco smoke: a report of the surgeon General. Atlanta: U.S: Department of Health and Human Services, Centers for Disease Control and Prevention, Coordinating Center for Health Promotion, National Center for Chronic Disease Prevention and Health Promotion, Office on Smoking and Health, 2006.

5 US Department of Health and Human Services. The health consequences of Smoking-50 years of progress: a report of the surgeon General. Atlanta, GA: US Department of Health and Human Services, Centers for Disease Control and Prevention, National Center for Chronic Disease Prevention and Health Promotion, Office on Smoking and Health, 2014.

6 Myers V, Shiloh S, Rosen L. Parental perceptions of children's exposure to tobacco smoke: development and validation of a new measure. BMC Public Health 2018;18:1031.

7 Benowitz NL. Cotinine as a biomarker of environmental tobacco smoke exposure. Epidemiol Rev 1996;18:188-204.

8 Walton K, Wang TW, Schauer GL, et al. State-Specific Prevalence of Quit Attempts Among Adult Cigarette Smokers - United States, 2011-2017. MMWR Morb Mortal Wkly Rep 2019;68:621-6.

9 Ashford KB, Hahn E, Hall L, et al. Measuring prenatal secondhand smoke exposure in mother-baby couplets. Nicotine Tob Res 2010;12:127-35.

$10 \mathrm{Kim} \mathrm{S}$. Overview of cotinine cutoff values for smoking status classification. Int J Environ Res Public Health 2016:13:1236.

11 Benowitz NL, Bernert JT, Caraballo RS, et al. Optimal serum cotinine levels for distinguishing cigarette smokers and nonsmokers within different racial/ethnic groups in the United States between 1999 and 2004. Am J Epidemiol 2009;169:236-48.

12 Dempsey DA, Meyers MJ, Oh SS, et al. Determination of tobacco smoke exposure by plasma cotinine levels in infants and children attending urban public hospital clinics. Arch Pediatr Adolesc Med 2012;166:851-6.

13 de Chazeron I, Llorca P-M, Ughetto S, et al. Occult maternal exposure to environmental tobacco smoke exposure. Tob Control 2007:16:64-5.

$14 \mathrm{Kim}$ Y, Choi Y-J, Oh S-W, et al. Discrepancy between self-reported and Urine-Cotinine verified smoking status among Korean male adults: analysis of health Check-Up data from a single private hospital. Korean J Fam Med 2016;37:171-6.

15 Pärna K, Rahu M, Youngman LD, et al. Self-Reported and serum cotinine-validated smoking in pregnant women in Estonia. Matern Child Health J 2005;9:385-92.

16 Max W, Sung H-Y, Shi Y. Who is exposed to secondhand smoke? self-reported and serum cotinine measured exposure in the U.S. 1999-2006. Int J Environ Res Public Health 2009;6:1633-48.

17 Dove MS, Dockery DW, Connolly GN. Smoke-free air laws and secondhand smoke exposure among nonsmoking youth. Pediatrics 2010;126:80-7.

18 Shenassa ED, Rossen LM, Cohen J, et al. Income inequality and US children's Secondhand smoke exposure: distinct associations by Race-Ethnicity. Nicotine Tob Res 2017:19:1292-9.

19 Bernert JT, Jacob P, Holiday DB, et al. Interlaboratory comparability of serum cotinine measurements at smoker and nonsmoker concentration levels: a round-robin study. Nicotine Tob Res 2009;11:1458-66. 
20 Braun JM, Kalloo G, Chen A, et al. Cohort profile: the health outcomes and measures of the environment (home) study. Int $J$ Epidemiol 2017;46:24.

21 Tsai J, Homa DM, Gentzke AS, et al. Exposure to Secondhand Smoke Among Nonsmokers - United States, 1988-2014. MMWR Morb Mortal Wkly Rep 2018;67:1342-6.

22 Thaqi A, Franke K, Merkel G, et al. Biomarkers of exposure to passive smoking of school children: frequency and determinants. Indoor Air 2005;15:302-10.

23 Woodward A, Al-Delaimy W. Measures of exposure to environmental tobacco smoke: validity, precision, and relevance. Ann N Y Acad Sci 1999;895:156-72.

24 Marano C, Schober SE, Brody DJ, et al. Secondhand tobacco smoke exposure among children and adolescents: United States, 20032006. Pediatrics 2009;124:1299-305.

25 Bowker K, Lewis S, Coleman T, et al. Changes in the rate of nicotine metabolism across pregnancy: a longitudinal study. Addiction 2015;110:1827-32.

26 Puig C, Garcia-Algar O, Monleon T, et al. A longitudinal study of environmental tobacco smoke exposure in children: parental self reports versus age dependent biomarkers. BMC Public Health 2008;8:47.

27 Aurrekoetxea JJ, Murcia M, Rebagliato M, et al. Secondhand smoke exposure in 4-year-old children in Spain: sources, associated factors and urinary cotinine. Environ Res 2016;145:116-25.

28 Jain RB. Exposure to second hand smoke at home and work among nonsmokers. Chemosphere 2015;135:225-32.

29 Okoli CTC, Kelly T, Hahn EJ. Secondhand smoke and nicotine exposure: a brief review. Addict Behav 2007;32:1977-88.

30 Polanska K, Krol A, Merecz-Kot D, et al. Environmental tobacco smoke exposure during pregnancy and child neurodevelopment. Int J Environ Res Public Health 2017;14:796.

31 Spanier AJ, Kahn RS, Xu Y, et al. Comparison of biomarkers and parent report of tobacco exposure to predict wheeze. J Pediatr 2011;159:776-82.

32 Martínez-Sánchez JM, Sureda X, Fu M, et al. Secondhand smoke exposure at home: assessment by biomarkers and airborne markers. Environ Res 2014;133:111-6. 